Article

\title{
Quelles capacités des traceurs GPS à rendre compte des pratiques de mobilité quotidienne ? Une application sur des territoires de faible densité en Indre et Loire (37, France)
}

\author{
Hervé Baptiste ${ }^{1 凶}$, Benoît Feildel ${ }^{2}$, Marie Huyghe ${ }^{3}$ \\ ${ }^{1}$ UMR 7324 CNRS-CITERES, Université de Tours, France \\ 2 UMR 6590 CNRS-ESO, Université Rennes 2, France \\ ${ }^{3}$ UMR 7324 CNRS-CITERES, Université de Tours, France
}

\begin{abstract}
Résumé. L'utilité des matériels et données associées de géolocalisation (loggersou traceurs-GPS, Smartphone) pour les recherches et enquêtes sur les mobilités quotidiennes ainsi que les limites de ces méthodes sont largement reconnues, et ont fait l'objet de nombreux articles à visée méthodologique. Notre article contribue à ces connaissances méthodologiques en apportant des éléments d'une part sur le protocole de traitement des données mis en œuvre au cours de la recherche, dont la littérature montre qu'il est encore en évolution suivant les équipes de recherche ; d'autre part sur les indicateurs d'analyse (statistique, territoriale, temporelle et cartographique) que nous avons développés, et des pistes de développements ultérieurs.

Mots clés : Suivi par GPS ; mobilité ; ruralité ; méthodologie d'enquête.
\end{abstract}

\section{Introduction : contexte de la recherche et problématique}

L'évolution du rapport à l'espace et au temps des modes d'habiter et des pratiques de mobilité individuelles, "complexes, variées, aléatoires, zigzagantes » (Gwiazdzinski et Drevon, 2014) appelle de nouveaux outils d'analyse et de représentation. Le

\section{CORRESPONDANCE :}

35 Allée Ferdinand de Lesseps, 37200 Tours, France.

@ herve.baptiste@univ-tours.fr (H. B.), benoit.feildel@univrennes2.fr (B. F.), https:/ / mariehuyghe. jimdo.com/, huyghe.marie@gmail.com (M. H.)

\section{HISTOIRE DE L'ARTICLE :}

Reçu : 27 septembre 2018

Reçu en forme révisée : 5 octobre 2018

Accepté : 7 octobre 2018

Disponible en ligne : 10 Octobre 2018 
développement d'outils de géolocalisation tels que les logger-GPS, tracker-GPS ou applications smartphone, leur perfectionnement progressif (en termes d'autonomie et de capacité de mémoire) et leur facilité d'accès croissante (grâce à des prix accessibles et une miniaturisation des appareils) a permis de renouveler les méthodologies d'enquêtes sur la mobilité et les déplacements. Depuis une quinzaine d'années en France, ces méthodes de collecte de données sont expérimentées dans de nombreux programmes de recherche et utilisées dans le cadre d'Enquêtes Ménages Déplacements (EMD) ou de l'Enquête Nationale Transports et Déplacements de 2008 (pour un recensement détaillé, voir Gwiazdzinski et Drevon, 2014, p.7).

L'utilité de ces outils de géolocalisation, largement reconnue, est de plusieurs ordres :

- les données recueillies sont précises, en termes de localisation des arrêts, d'itinéraires parcourus, de chaînage de déplacements, de temporalité des déplacements (horaires d'arrivée et de départ, temps sur place), de vitesse, etc. Les trackers-GPS et les applications sur smartphone (mais pas les loggers-GPS les plus simples) permettent de caractériser plus finement les déplacements : selon les besoins de l'enquête ou de la recherche, les enquêtés peuvent être invités à préciser le mode de transport utilisé, le motif du déplacement, son caractère régulier ou non, la présence d'autres personnes dans le véhicule, qui finance le déplacement, etc.

- les données enregistrées par les appareils sont objectives: contrairement aux données produites lors des EMD traditionnelles, les données acquises par GPS ne « ressortent pas du filtre subjectif des enquêtés » (Depeau et Quesseveur, 2014), elles sont donc fiables et a priorit représentatives des pratiques réelles des enquêtés.

- les données peuvent être recueillies sur des pas de temps longs, allant de plusieurs jours à plusieurs mois: ceci permet notamment de discerner les activités et déplacements routiniers des plus exceptionnels. Les protocoles mis en œuvre dans les enquêtes de mobilité traditionnelles (qui font appel à la mémoire des enquêtés) ou les carnets de pratiques (méthodes lourdes pour l'enquêté, à qui il est demandé de retranscrire en détail l'ensemble de ses déplacements) restreignent les durées d'enquêtes à la journée, voire à la semaine.

La littérature (notamment Drevon et al, 2014) insiste sur l'intérêt des GPS pour les enquêtes de mobilité, qui permettent de compléter et d'améliorer les protocoles d'enquêtes traditionnels ou de valider les données acquises. En particulier, les outils-GPS permettent de limiter l'effort de mémoire imposé aux personnes enquêtées, d'identifier des informations manquantes parfois oubliées par les individus lors des entretiens (comme certains arrêts intermédiaires ou les

\footnotetext{
${ }^{4}$ Les enquêtés peuvent oublier, sciemment ou non, d'emporter leur GPS lors de leurs déplacements ; les traces obtenues ne seront donc pas parfaitement représentatives de leurs pratiques réelles.
} 
déplacements les plus courts, pour rejoindre son véhicule ou en rabattement vers un transport en commun par exemple), et enfin de limiter les durées des interviews. En raison de limites techniques, il est aujourd'hui difficile de réaliser des enquêtes de mobilité avec des GPS uniquement (Nguyen, 2012): des interviews même courtes sont encore nécessaires pour faire parler certaines informations «muettes", en particulier les motifs de déplacement (sauf à utiliser des appareils d'enregistrement qui permettent de les renseigner en direct).

Une fois les données obtenues, qu'en faire? On trouve dans la littérature des analyses de traces-GPS selon les caractéristiques territoriales, les contextes spatiaux (Depeau et Quevesseur, 2014, ont analysé l'influence de "l'imprégnation verte » ou de «l'attractivité sociale » sur les pratiques de mobilité d'enfants), ou encore selon les caractéristiques sociodémographiques des individus (Gwiazdzinski, 2006, s'est intéressé à l'influence du genre sur la taille de l'espace de vie). On trouve également des analyses temporelles des traces GPS, en termes de temps de déplacement et de durées d'activités, de comparaisons semaine-week-end ou de temps d'immobilité par exemple (Gwiazdzinski et Klein, 2014 ; Aguiléra et al, 2016).

Enfin, les données produites par les GPS peuvent donner lieu à la production « d'objets-GPS » (cartographies animées ou non, vidéos géo-référencées - Drevon et al, 2015) qui constituent des «embrayeurs de discours » (Feildel, 2014) et permettent ainsi de compléter le volet qualitatif de la méthode. Dans le programme de recherche Périvia (Martouzet et al, 2012), les traces-GPS ont été soumises aux enquêtés afin de réactiver leurs discours et de comprendre les raisons prévalant à leurs choix (choix d'itinéraire, de mode de transport, etc.) ; Drevon et al (2015) ont quant à eux utilisé des vidéos géo-référencées pour amorcer une discussion avec les enquêtés sur les «barrières au déplacement ». Les «objets-GPS », en particulier les représentations dynamiques et interactives, sont des outils pédagogiques particulièrement valorisables pour sensibiliser les acteurs du territoire (Daoud et al, 2009) et imaginer des politiques publiques adaptées.

Les limites des méthodes à base de GPS sont également largement connues. Elles sont en particulier d'ordres techniques et technologiques, liées à l'appareil de mesure et aux logiciels de traitement des données. Sont ainsi mentionnées dans la littérature des difficultés de réception du GPS (dans les tunnels, les «canyons urbains ») et production de points «parasites " (aussi qualifiés de «erratiques » ou "fantômes ») autour des lieux d'arrêt ; l'insuffisance de la capacité d'enregistrement ou de l'autonomie de l'appareil ; la perte de données pendant l'enregistrement ou au moment de leur transfert. Les capacités des appareils ne cessent de s'améliorer, notamment en termes d'autonomie et de mémoire; on note par ailleurs dans les projets les plus récents une utilisation croissante d'appareils plus perfectionnés (trackers ou applications sur smartphone) ou de données issues des Big Data. 
Sont également très fréquemment évoquées les difficultés de traitement des données, en particulier liées au filtrage (suppression des points et parcours fantômes) ou à l'identification des points d'arrêt. Ces difficultés sont à relier au caractère encore relativement artisanal et très évolutif des protocoles de traitement: d'après la littérature, chaque programme de recherche (ou laboratoire de recherche) semble développer indépendamment son propre protocole ou logiciel de traitement. Par exemple, Poirier et al (2015) ont identifié les points d'arrêts de manière manuelle ${ }^{5}$, méthode laborieuse, chronophage, et non exempte d'erreurs d'interprétations; Depeau et Quesseveur (2014, p.50) évoquent «la mise au point de programmes de formatage..." et "des traitements qui sont au stade de l'exploration mais ne sont ni formalisés, ni stabilisés »; enfin, Nguyen (2012, p.41) cite son logiciel "systèmeexpert » G-TO-MAP.

Les difficultés de la méthode ont également trait aux enquêtés, qui doivent porter l'appareil pendant plusieurs heures à plusieurs mois. Ce peut être des difficultés logistiques au moment des enregistrements : échange de l'appareil au sein du ménage, oubli (réel ou volontaire) de l'appareil pendant des déplacements, oubli de rechargement. Par ailleurs, alors que l'accent est mis au moment du dépôt de l'appareil sur l'importance de ne pas modifier ses habitudes de déplacement pendant la période d'enregistrement, certains enquêtés tendent à limiter leurs trajets ou à adopter des modes de transports "plus vertueux » (modes actifs notamment) pour « plaire à l'enquêteur» (Huyghe et al, 2014, p.103).

Enfin, les méthodes basées sur de la géolocalisation se heurtent à des limites éthiques, malgré un encadrement strict par la CNIL (en France) : les données-GPS permettent d'identifier facilement les lieux de résidence et d'emploi, et par là l'identité des enquêtés, voire leurs préférences religieuses ou politiques (via les lieux de culte ou les lieux de regroupements politiques pratiqués); l'analyse temporelle des traces-GPS donne également des indications sur les heures et lieux de présence des enquêtés, qui pourraient par exemple être utilisées pour cambrioler les domiciles (Primault et al, 2015). Garantir l'anonymat des enquêtés requiert d'altérer les traces (de flouter l'itinéraire précis, de brouiller ou rendre impossible la localisation des points d'arrêt, de modifier les horaires), mais risque de rendre les données inutilisables. Ces questions éthiques peuvent provoquer une frilosité par rapport au protocole chez certains publics; cependant, plusieurs expériences menées auprès d'enfants (et leurs parents) et d'adolescents montrent que le caractère ludique de ces technologies peut au contraire agir comme un facilitateur, et permettre de mobiliser plus facilement ces populations (Gwiazdzinski, 2008).

\footnotetext{
5 A partir des représentations cartographiques des données, ils ont localisé les amas de points, qui correspondent à un point d'arrêt ; dans la base de données, ils ont ensuite identifié les données associées à l'amas de point : le point d'arrêt est alors « borné » dans le temps, en identifiant le premier et le dernier point présentant des caractéristiques similaires en vitesse et en distance au point précédent.
} 
L'article que nous proposons se base sur des données-GPS produites en 2012 et 2014, au cours de deux programmes de recherche sur la mobilité quotidienne dans les territoires de faible densitét. Nous ne reviendrons pas ici sur les conclusions scientifiques des programmes, qui portent essentiellement sur une meilleure connaissance et compréhension des pratiques de mobilité individuelle dans les territoires ruraux et périurbains, des raisons prévalant aux choix des ménages (en termes de lieux d'activité, de mode de transport, etc.) et des liens entre pratiques de mobilité et caractéristiques territoriales: nous renvoyons le lecteur aux rapports finaux des programmes (Baptiste et al, 2013 ; Aguiléra et al, 2016). relatifs à :

Les recherches poursuivaient également des objectifs méthodologiques,

- la construction de données, leur traitement, leurs apports et limites quant à leur capacité à compléter la connaissance de mobilités acquises par les entretiens semidirectifs : comment produire des données ? à quoi servent-elles ?

- la construction et la mise en œuvre d'indicateurs pour caractériser les liens entre mobilité et dynamique des territoires : comment analyser les données produites ?

- l'efficacité et l'efficience des modes de représentation des résultats : quelles limites à la méthode?

- la complémentarité/le potentiel de substitution de la méthode-GPS avec d'autres méthodes d'analyse des mobilités.

Le volume 28 de la revue Netcom (Drevon et Gwiazdzinski, 2014) est particulièrement riche sur la question des méthodes et outils au service de l'observation, l'analyse et la représentation des pratiques de mobilité.

Nous proposons ainsi dans notre article de discuter deux points en particulier :

- le protocole de traitement mis en œuvre puisque, comme mentionné précédemment, on observe encore des tâtonnements dans les protocoles testés par les différentes équipes de recherche ;

- les indicateurs d'analyse (statistique, territoriale, temporelle et cartographique) que nous avons développés: nous indiquons à chaque fois les points qu'il convient d'améliorer ou de développer plus en avant ;

Nous reviendrons sur le volet des limites uniquement lorsqu'elles n'ont pas ou peu été analysées ou décrites dans la littérature.

\footnotetext{
${ }^{6}$ Il s'agit des programmes MOUR (Mobilité et Urbanisme Rural - 2011-2013) et MOBITER (Mobilité et dynamique des territoires ruraux - 2013-2015), menés au laboratoire CITERES de l'Université de Tours en partenariat avec (pour le premier) le Parc Naturel Régional Loire Anjou Touraine et (pour le second) le laboratoire LVMT (Université de Marne la Vallée).
} 


\section{Présentation du matériau de recherche}

Notre article s'appuie sur l'analyse d'une base de 57 tracés-GPS, obtenus par l'enregistrement des déplacements de 57 individus pendant (plus ou moins) une semaine, lors de deux campagnes d'enquête en 2012 (programme MOUR) et 2014 (programme MOBITER) ${ }^{7}$. Nous avons choisi de mutualiser l'ensemble des traces dont nous disposions afin de construire un échantillon d'analyse suffisamment consequent ; ce faisant, comme exposé ci-après, nous nous sommes exposés à des difficultés d'analyse.

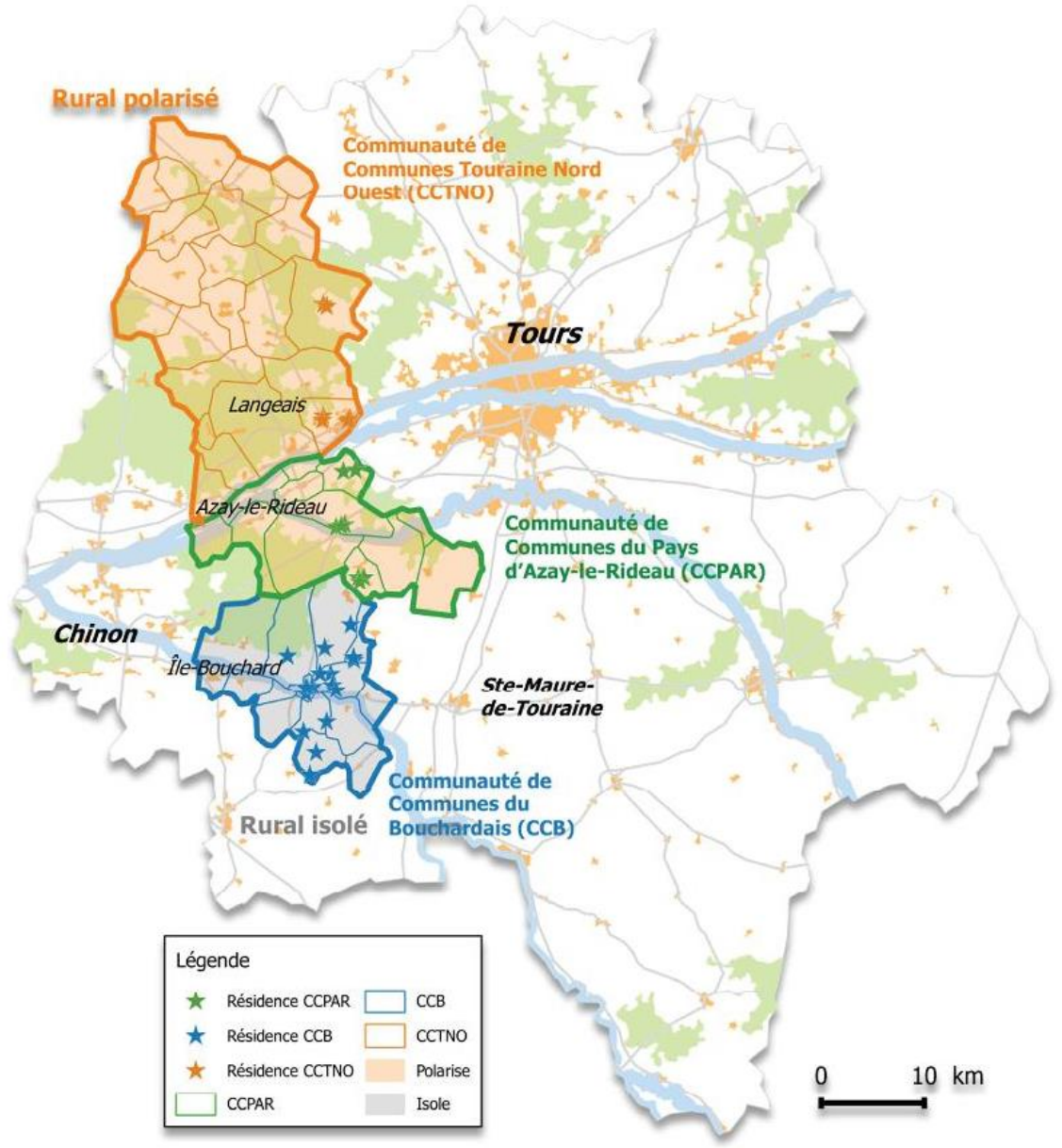

Carte 1 : Lieux de résidence des volontaires au suivi-GPS dans les territoires d'étude - B. Feildel, 2015

\footnotetext{
${ }^{7}$ L'analyse de l'ensemble des données a été réalisée au cours du programme MOBITER, ainsi que par des étudiants de $4^{\text {ème }}$ et $5^{\text {ème }}$ année d'ingénieur en Génie de l'Aménagement et de l'Environnement de Polytech Tours (Université de Tours), qui ont notamment réalisé certaines des cartes présentées dans cet article.
} 
Les individus enquêtés résidaient dans trois territoires d'étude dans le département d'Indre-et-Loire (France) ${ }^{8}$ : deux territoires qualifiés de « rural polarisé » situés à une vingtaine de kilomètres de Tours et soumis à l'influence du pôle urbain (au nord-ouest et ouest de Tours, en vert et orange sur la carte 1); un territoire " rural isolé » situé à une cinquantaine de kilomètres de Tours et moins soumis à son influence (au sud-ouest de Tours, en bleu sur la carte).

Pour une présentation détaillée du panel, nous renvoyons le lecteur au rapport final du programme MOBITER (Aguiléra et al, 2016, p.35); notons-en simplement quelques caractéristiques :

- équilibre du panel entre rural polarisé (29 enregistrements) et rural isolé (28 enregistrements) ;

- surreprésentation, par rapport aux moyennes nationales, des ménages avec enfants dans le rural polarisé (23 sur 29) et de la CSP « employés » dans le rural isolé (12 sur 29).

Nous avons eu globalement peu de difficultés à identifier des volontaires pour participer à la campagne d'enregistrement et aboutir à un panel relativement équilibré en termes d'âge ou de sexe notamment. On peut définir deux profils principaux parmi les personnes ayant refusé de participer ou choisi d'interrompre l'enregistrement en cours de semaine :

- des personnes méfiantes face à la méthodologie d'enquête, notamment en raison d'un manque d'habitude des appareils d'enregistrement : ce sont notamment des femmes retraitées et/ou conjointes d'interviewés qui n'avaient pas assisté à la présentation de la recherche; un enquêté a également mentionné son refus «de servir de cobaye » et "d'être fliqué » (Huyghe et al, 2014, p.103) ;

- des actifs très mobiles qui se déplaçaient souvent et longtemps au cours de leur journée de travail (agriculteurs notamment) : ils exprimaient un manque d'envie à transporter l'appareil toute la journée au risque de le perdre ou le casser.

L'argument « je me déplace peu», «mes déplacements ne sont pas intéressants » a plusieurs fois été avancé par des interviewés peu motivés : expliquer que tous les profils de mobiles étaient intéressants pour la recherche n'a pas toujours suffi à convaincre ces personnes à accepter le GPS.

Les appareils d'enregistrements sont des traceurs-GPS i-gotU : leur capacité d'autonomie et d'enregistrement relativement limitée (ils devaient être rechargés $4 \mathrm{~h}$ toutes les nuits) en fait des appareils aujourd'hui dépassés. Néanmoins, nous avons choisi d'utiliser le matériel dont nous disposions au laboratoire.

\footnotetext{
${ }^{8} \mathrm{Cf}$. Annexe pour une présentation succincte de l'organisation administrative du territoire français.
} 
Nous détaillons ci-dessous quelques éléments de paramétrage des appareils et de protocole d'enregistrement, utiles à notre analyse. Suite à des dysfonctionnements observés lors de la première campagne d'enregistrement (mémoire du GPS pleine qui a conduit à l'interruption de l'enregistrement), nous avons modifié le paramétrage des appareils, en particulier diminué la fréquence d'enregistrement.

\section{Paramétrage des appareils :}

Concernant la fréquence d'enregistrement, elle varie en fonction de la vitesse de déplacement de l'individu (intervalle de 1 ou 3 secondes pour les déplacements effectués à vitesse inférieure à $30 \mathrm{~km} / \mathrm{h}$ - à pied ou à vélo; intervalle de 3 ou 5 secondes pour les déplacements effectués à une vitesse supérieure à $30 \mathrm{~km} / \mathrm{h}$ - mode de transport motorisé). Ce choix initial a été justifié par la durée totale d'enregistrement visée (une semaine par individu), la capacité de mémoire des appareils et notre souhait de limiter au maximum, pour les enquêtés, les manipulations lors de la semaine d'enregistrement.

Quant à la plage d'enregistrement, elle varie selon que l'on est en semaine/week-end (de $6 \mathrm{~h}$ à $22 \mathrm{~h}$ en semaine, en continu le week-end); ce faisant, des déplacements nocturnes éventuels en semaine seront exclus de nos bases de données.

Enfin les enregistrements ont été réalisées (sauf exceptions ou dysfonctionnements) sur une semaine complète: ils incluent ainsi les déplacements récurrents (domicile-travail) et les déplacements moins systématiques du week-end, mais laissent potentiellement sous silence les déplacements effectués à des fréquences plus exceptionnelles (mensuelles, annuelles...) ou, au contraire, survalorisent un déplacement exceptionnel réalisé durant la période d'enregistrement.

\section{Protocole d'enregistrement:}

Notons le caractère asynchrone des enregistrements (au cours d'une même campagne d'enregistrement) : compte tenu de la complexité à mobiliser des individus volontaires (notamment par le caractère intrusif de la méthode), a fortiori pendant une semaine «type » (hors vacances scolaires pour les ménages avec enfant par exemple), les enregistrements ont été effectués autant que possible lors d'une même semaine, mais parfois lors de semaines différentes: cela induit notamment des différences dans les conditions météorologiques lors des enregistrements.

Enfin, il s'agit de rester vigilant par rapport au comportement des individus suivis par un traceur GPS: nous avons observé ou fait l'hypothèse d'oublis (intentionnels ou non) des traceurs GPS à leur domicile, générant une «immobilité » erronée (pendant des journées entières par exemple) ; quelques individus suivis nous ont également fait part de changements intentionnels de pratiques de déplacements 
(modes actifs privilégiés, rationalisation de déplacements, etc.) lors de la période de suivi GPS. En conséquence, quelques enregistrements n'ont pas été considérés par la suite dans notre échantillon de travail.

Le déséquilibre observable entre le nombre total de points enregistrés dans le rural polarisé (3 406 882) et le rural isolé (1 604 759) s'explique par la différence de paramétrage de la fréquence $d^{\prime}$ 'enregistrement entre la $1^{\text {ère }}$ et la $2^{\text {nde }}$ campagne d'enregistrement : dans le 1 ${ }^{\text {er }}$ terrain investigué en 2014 (la CCPAR, rural polarisé), la fréquence était 3 fois supérieure pour les déplacements lents, et quasiment 2 fois supérieure pour les déplacements rapides, à celle paramétrée pour le $2^{\text {nd }}$ terrain.

Tableau 1 : Notre échantillon de données est constitué comme suit :

\begin{tabular}{lrrr}
\hline & Rural polarisé & Rural isolé & TOTAL \\
\hline Nb de traces GPS (i.e. d'individus suivis) & 29 & 28 & 57 \\
Nb de points d'enregistrement & 3406882 & 1604759 & 5011641 \\
Nb de jours d'enregistrement & 218 & 198 & 416 \\
Nb de km parcourus & 15052 & 14142 & 29194 \\
Nb moyen de km parcourus par jour & 69.0 & 71.4 & 70.2 \\
\hline
\end{tabular}

Si cela rend impossible toute comparaison en valeur absolue entre les deux types de territoires, il n'en reste pas moins possible de traiter ensemble ces données en travaillant en proportion.

\section{Protocole de traitement des données}

Les données générées par les traceurs GPS sont importées dans le logiciel propriétaire des appareils (@tripPC) : on obtient un ensemble de points caractérisés par leurs coordonnées en latitude, longitude et altitude, leur date et horaire ; sont ajoutés pour chaque point les résultats de calculs de la vitesse, de la distance parcourue depuis le point précédent et de la distance cumulée depuis le premier point d'enregistrement de la journée.

La représentation de ces points «bruts» laisse apparaître des points erratiques, agglomérés sous forme de « pelotes » de points aberrants.

A titre exploratoire, un logiciel a ainsi été testé pour supprimer ces artefacts : GPS Track Editor, logiciel libre. Selon les éditeurs, l'algorithme utilisé estime «la cohérence » entre les points adjacents enregistrés et supprime ceux ne l'étant pas. Un premier test a été effectué avec une trace GPS de notre panel : 10107 points ont été identifiés comme points erratiques sur les 12859 points enregistrés au total, laissant seulement 2752 points réputés réels. A l'évaluation de la " cohérence » entre les points adjacents, ce dernier intègre donc vraisemblablement une procédure de simplification de la trace, par suppression de points intermédiaires sur des tronçons 
rectilignes par exemple. En raison de l'insuffisance d'informations sur l'algorithme déployé par le logiciel et du résultat de notre test, nous avons choisi d'utiliser le logiciel de traitement MobiRev, développé par B. Feildel (2014) en collaboration avec un élève ingénieur en informatique (cf. Serra, 2014); il identifie les points aberrants en comparant l'ensemble des points de la trace à des valeurs numériques renvoyant à des seuils fixés ou non par l'utilisateur, correspondant à des situations impossibles du point de vue des pratiques de mobilité des individus, en termes de vitesse instantanée, d'accélération ou de décélération.

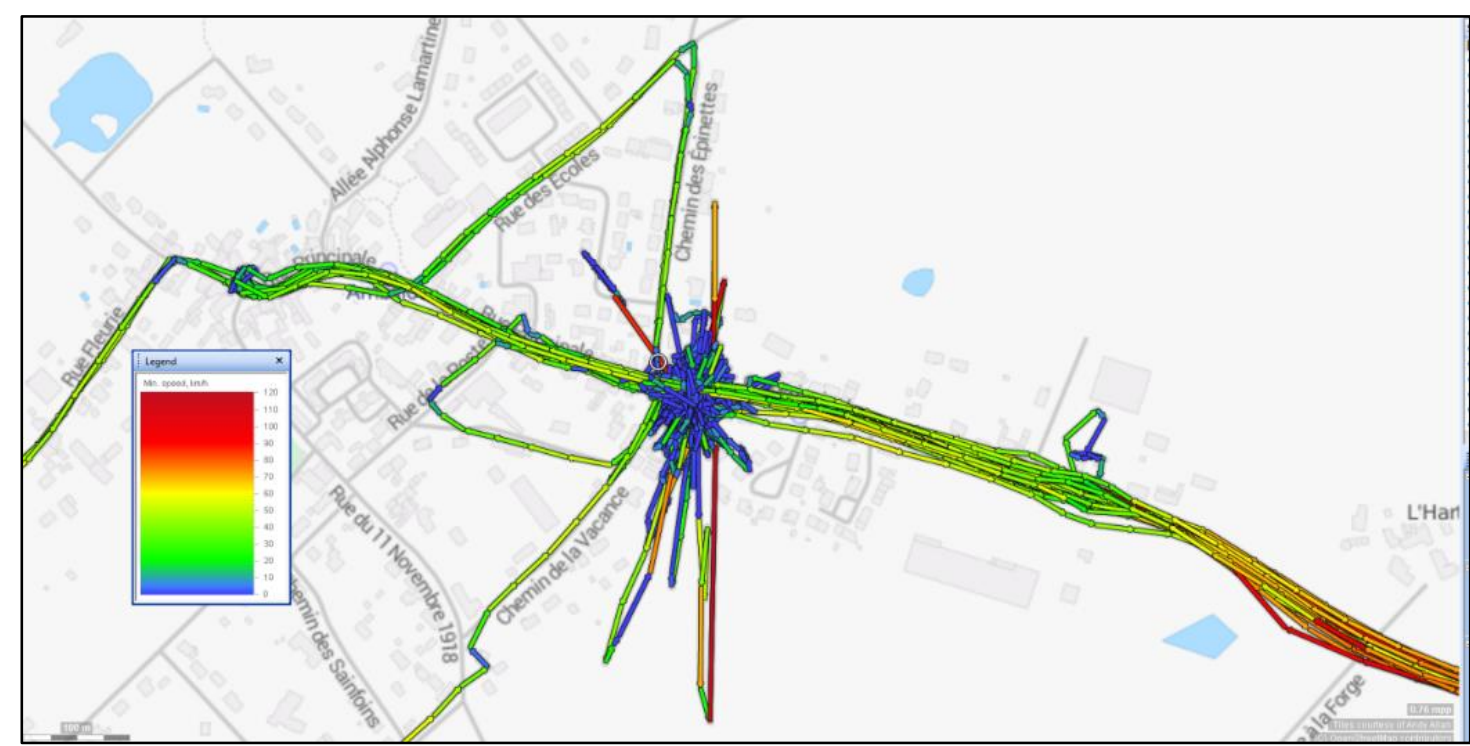

Figure 1 : Trace de M. B1 (CCTNO, du 25/09/2014 au 01/10/2014) - Vue de détail sur la localisation du domicile, points erratiques (logiciel GPS Track Editor)

Il faut ensuite identifier les points d'arrêt. Le développement du logiciel MobiRev a été l'occasion d'automatiser l'identification des points d'arrêt. L'algorithme de détection automatique des points d'arrêts a été implémenté selon une logique simple, pragmatique, basée sur l'analyse des angles formés par l'ensemble des vecteurs-vitesse, vecteur représentant la vitesse et la direction de l'objet mobile (ici le GPS) à un instant $t$, qui composent la trace. Dès lors que cet angle formé par les vecteurs-vitesse successifs s'avérait supérieur à un seuil de 90 degrés, et que le phénomène se répétait de façon consécutive un certain nombre de fois, il a été inféré que nous avions à faire à un ensemble continu de points erratiques, et donc à une situation d'immobilité assimilable à un point d'arrêt dans le déplacement de l'individu. La durée de ce point d'arrêt a ensuite été déterminée en comptabilisant le temps écoulé entre le premier et le dernier point de cet ensemble de points erratiques. 


\section{Construction d'indicateurs pour l'exploitation des données et représentation cartographique}

Revenons à présent sur les indicateurs développés pour notre analyse. Nous distinguons trois types $\mathrm{d}^{\prime}$ indicateurs :

- en 3.1. : des indicateurs synthétiques, issus des statistiques spatiales descriptives, pour lesquels les pratiques sont décrites uniquement par une distance ;

- en 3.2. : des indicateurs spatialisés d'inscription territoriale des mobilités, pour lesquels les pratiques sont décrites par des coordonnées de longitude et latitude ;

- en 3.3 : des indicateurs d'analyse temporelle ;

- en 3.4. : des indicateurs d'analyse cartographique.

\subsection{Indicateur de couverture spatiale}

\subsubsection{Distances cumulées aux lieux de résidence}

Cet indicateur permet de mesurer l'étendue du territoire investi (traversé ou habité) par les individus, le degré de centralité ou de dispersion de la structure complexe de leurs espaces de vie. Parce qu'il se base sur l'ensemble des points (points d'arrêts et points enregistrés pendant les déplacements), l'indicateur révèle indistinctement le mouvement et l'immobilité chez les individus.

\section{Lieu de résidence et distances cumulées}
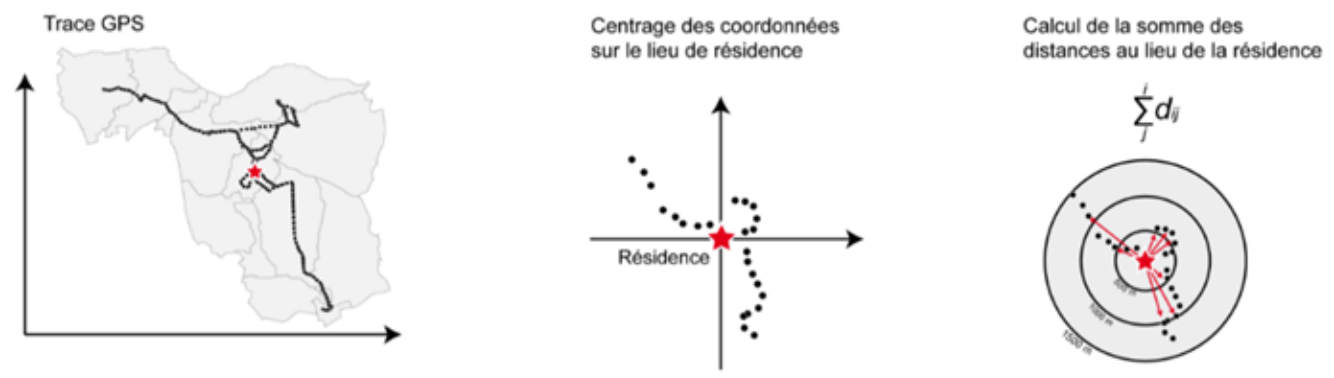

Figure 2 : Méthode de calcul de l'indicateur de distances cumulées au lieu de résidence - B.Feildel, 2015

Pour chaque trace récoltée par individu, la distance kilométrique à vol $d^{\prime}$ oiseau entre le domicile et chaque point d'enregistrement est calculée ${ }^{9}$ (cf. Figure 2)

\footnotetext{
${ }^{9}$ La distance entre le lieu de domicile (Latitude à l'origine et Longitude à l'origine) et chaque point enregistré (Latitude à destination et Longitude à destination) est définie par l'équation : $=A \operatorname{Cos}(\operatorname{Sin}$ (Radians(Latitude à l'origine))*Sin(Radians(Latitude à destination)) $+\operatorname{Cos}($ Radians(Latitude à l'origine))* ${ }^{*} \operatorname{Cos}($ Radians (Latitude à destination) $){ }^{*} \operatorname{Cos}($ Radians(Longitude à l'origine - Longitude à destination)))* ${ }^{*} 6371$.
} 
puis fait l'objet d'une sommation pour l'ensemble des individus d'un type de territoire rural (polarisé ou isolé). L'indicateur est in fine une mesure de la dispersion des points par rapport aux lieux de domicile des individus.

Les données calculées sont ensuite rapportées au nombre de jours d'enregistrement, puis segmentées par classes de distance au lieu de résidence.

Pour pallier les paramétrages différents des appareils au cours des deux campagnes d'enregistrement, les nombres de points enregistrés par classes de distance sont enfin rapportés au nombre total de points enregistrés; on obtient des «proportions de points enregistrés par plage de distance».

Une première exploration de cet indicateur montrait une très forte surreprésentation du nombre de points dans la plage de distance inférieure à $50 \mathrm{~m} \mathrm{du}$ lieu de domicile. Un examen de la base de données a montré qu'outre les points enregistrés au domicile, les points erratiques, générés notamment par une captation plus difficile du signal GPS lorsque la personne était dans son domicile, étaient massivement dispersés dans un rayon inférieur à $50 \mathrm{~m}$ du domicile. Si ces résultats traduisaient certes des durées habituelles d'immobilité prolongées au domicile, ils « écrasaient » et faussaient en termes de représentation les résultats graphiques des autres plages de distance.

Le résultat graphique ci-après (cf. Figure 3), négligeant donc la plage assimilée au séjour des personnes dans leur lieu de résidence, met en évidence des différences très nettes entre les territoires ruraux polarisés et isolés.

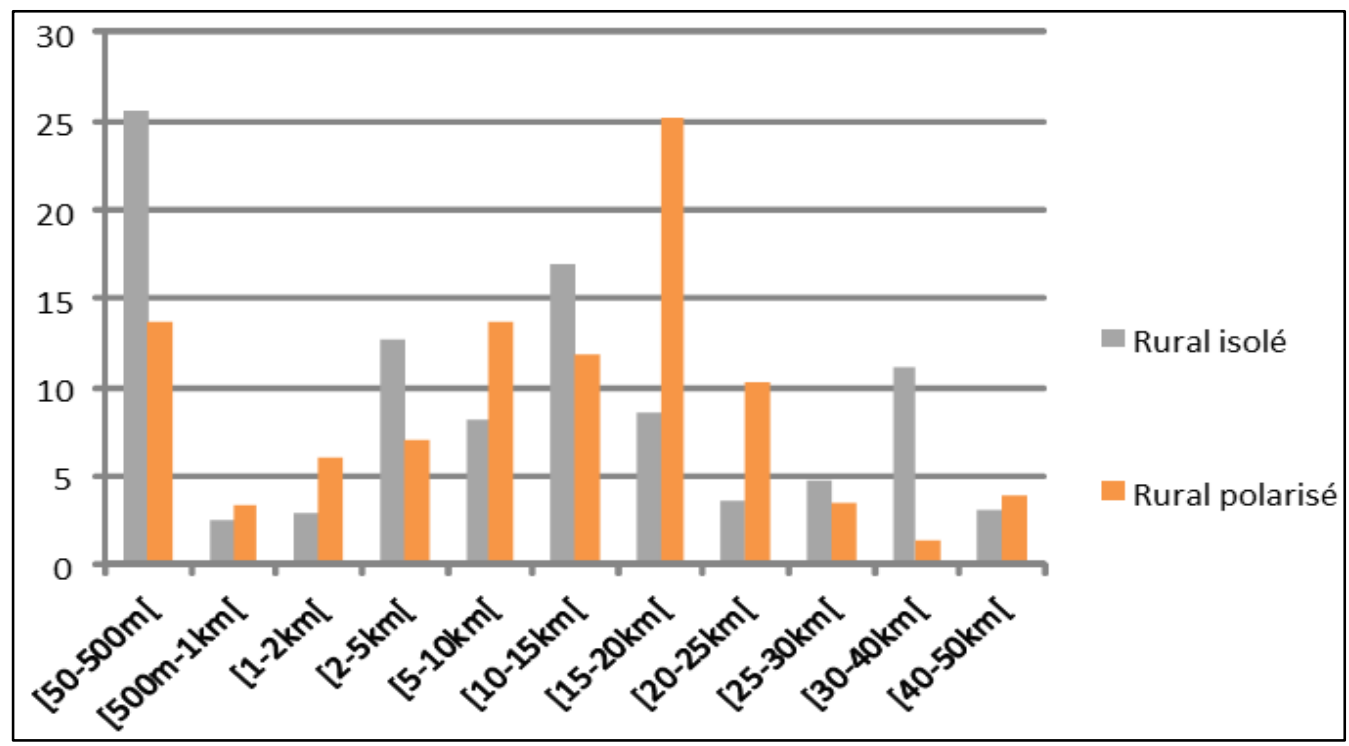

Figure 3 : Proportion de points par plage de distance (de $50 \mathrm{~m}$ à $50 \mathrm{~km}$ du domicile), selon le type de territoire rural étudié (polarisé ou isolé) - H. Baptiste, 2015 


\section{Limites de la méthode mobilisée :}

Alors que les GPS sont censés pallier des insuffisances des enquêtes traditionnelles, en particulier en termes d'identification des déplacements courts/déplacements à pied, notre méthode n'est pas exempte de limites sur ce point. En effet, nous rappelons que les traceurs GPS ont été paramétrés pour enregistrer des points à des fréquences variables selon la vitesse de déplacement du volontaire (fréquence plus élevée pour des déplacements lents ; fréquence plus faible pour des déplacements rapides).

Dès lors, comment interpréter le nombre plus élevé de points dans la classe 50-500m ? S'explique-t-il par des déplacements courts réalisés à pied (avec une fréquence d'enregistrement plus élevée) ou par des déplacements courts réalisés en voiture (avec une fréquence d'enregistrement plus faible), mais plus fréquemment ?

La méthode que nous avons mise en œuvre ne nous permet pas de répondre : il faudrait réitérer ce protocole sur un nouvel échantillon à constituer, mais cette foisci avec une fréquence d'enregistrement constante pour l'ensemble des déplacements, indépendamment de leur vitesse.

\subsubsection{Distances cumulées à un point central}

En procédant à une mesure de distance standard (Gerber et Carpentier, 2012), ce deuxième indicateur permet de rendre compte de l'éloignement de chacun des points du relevé GPS par rapport au « centre moyen » (ou point central), au sens statistique du terme (point virtuel, qui ne correspond plus au lieu de résidence) de la trace (cf. Figure 4): nous obtenons ainsi une mesure synthétique de la centralité et de la dispersion représentative de la structure complexe des espaces de vie des individus. Ce centre moyen $\bar{P}$, qui minimise la distance aux autres points de la trace, est obtenu en effectuant la moyenne des coordonnées de l'ensemble des points enregistrés.

La mesure de la distance standard consiste alors à calculer la distance moyenne de chaque point à ce point central $\bar{P}$. La dispersion (ou coefficient de variation $C v$ ) résulte alors $d u$ rapport entre l'écart-type $\delta$ et la moyenne $\mu$ des distances au point central.

La mesure de la dispersion relative des traces depuis le point central apporte un éclairage complémentaire par rapport à la mesure des distances cumulées au lieu de résidence. Les résultats montrent notamment que les plus forts coefficients de dispersion $\mathrm{Cv}$ s'expriment dans le rural isolé (où les points se dispersent jusqu'à une valeur de 2,5 à 3,5 autour du point central); ils montrent également que les mobilités sont globalement plus concentrées dans le rural isolé (avec des coefficients resserrés autour des valeurs entre 0,5 et 1,5 ) et globalement plus dispersées dans le polarisé (avec un plus grand nombre de coefficients de variation compris entre les plages de 
valeurs de 0 à 2,5 .

Si les résultats issus de cette mesure de dispersion méritent d'être approfondis et étayés par un plus grand nombre de relevés GPS, il n'en demeure pas moins que cet indicateur permet d'entrevoir un possible « effet de lieu » (Bourdieu, 1993) propre aux territoires ruraux isolés et polarisés, autrement dit une différenciation des pratiques spatiales corrélative au type de territoire.

\section{Mesure de la dispersion relative des traces depuis le point central}

Point central : point théorique qui minimise la distance aux autres points de la trace GPS

$$
\bar{P}(\text { Lat }, \text { Long })=\frac{1}{n_{i}} \sum_{P i}^{n}(\text { Lat }, \text { Long })
$$

Coefficient de variation : rapport entre l'écart-type et la moyenne des distances au lieu central

$$
\delta_{A}=\sqrt{\frac{\sum d^{2}}{n}} \quad C v=\frac{\delta}{\mu}
$$

Des mobilités plus dispersées dans le rural polarisé ; un coefficient de variation moins élevé dans le rural isolé.

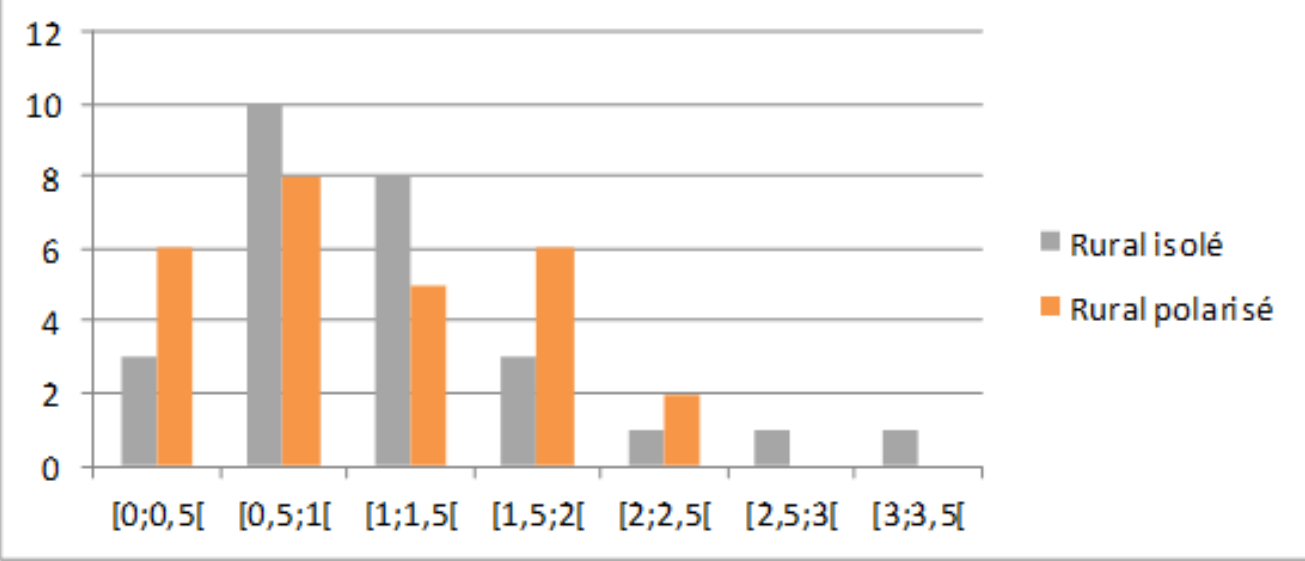

Figure 4 : Mesure et résultats de la dispersion $(C v)$ de chacune des traces selon le type de territoire étudié : des mobilités plus dispersées dans le rural polarisé, un coefficient de variation moins élevé dans le rural isolé - H. Baptiste, B. Feildel, 2015

Dans le rural isolé, la majorité des points d'enregistrement semble se concentrer autour d'un point central, mais certains demeurent relativement éloignés de celui-ci : les déplacements des ménages se réalisent majoritairement à proximité du lieu central, mais pas exclusivement, puisque certains se font dans des classes de distances éloignées. Dans le rural polarisé, les coefficients de dispersion varient de façon sensiblement plus importante : les déplacements des ménages se réalisent dans un espace plus diffus que dans l'isolé, moins concentré autour du point central. 
Aussi, nous faisons l'hypothèse que l'espace rural isolé a une influence plus grande que l'espace rural polarisé sur l'organisation sociale dans ces espaces et la structuration des mobilités habitantes.

\subsubsection{Dispersion des points d'arrêt par rapport au lieu de domicile}

A la différence des deux indicateurs précédemment exposés, ce troisième tient compte uniquement des points d'arrêts identifiés : il permet donc de caractériser l'étendue des territoires réellement pratiqués (pour différents motifs d'activités) par les ménages.

Nous ne reviendrons pas sur sa méthodologie de construction, très similaire à celle présentée précédemment (cf. 3.1.2). Notons simplement que les résultats obtenus sont relativement similaires à ceux basés sur l'indicateur des distances cumulées.

D'un point de vue méthodologique, il semblerait donc que l'analyse de l'ensemble des points d'enregistrement suffise également pour caractériser l'étendue spatiale des lieux pratiqués par les individus. Si cette hypothèse est validée, cela permettrait de simplifier les phases de traitement, puisqu'il ne serait plus nécessaire de construire une base de données des points d'arrêts. En revanche, cette simplification se traduit en retour par une masse beaucoup plus importante de données à traiter, potentiellement limitante selon la capacité des ordinateurs.

\section{Indicateur spatialisé}

Les résultats issus des indicateurs précédents s'avèrent intéressants pour mesurer l'étendue des territoires vécus et/ou parcourus par les volontaires. Toutefois, une question reste posée : quels types de territoires sont prioritairement investis par les individus?

Comme Depeau \& Quevesseur (2014), nous avons souhaité analyser les pratiques de mobilité des ménages au regard des caractéristiques des territoires qu'ils fréquentent. L'indicateur suivant vise ainsi à croiser la localisation des points d'arrêt (selon une mesure de leur densité par commune fréquentée) et le type de territoire, selon le zonage en aires urbaines (ZAU 2010 de l'INSEE).

En termes de résultats (cf. Aguiléra et al, 2016 p. 53 pour des résultats détaillés), notons simplement qu'ils convergent avec ceux obtenus par les indicateurs précédents: ils mettent notamment en évidence une densité de points d'arrêts importante dans les territoires de proximité, montrant ainsi un investissement significatif des individus dans le local, pour les habitants des deux types de territoire, ainsi qu'une densité de points d'arrêt dans le grand pôle beaucoup plus importante pour les habitants du rural polarisé que pour ceux de l'isolé. 
$\mathrm{Au}$ niveau de la méthodologie déployée, tous les points d'arrêts ont été considérés pour tester l'indicateur, afin d'éprouver ses capacités à rendre compte du territoire vécu par les individus dans son ensemble: en conséquence, les représentations cartographiques par cercles de taille proportionnelle tendent à minimiser le poids des lieux fréquentés qui sont associés à des motifs d'activité hors du domicile. Aussi, la méthode pourrait être développée en excluant du calcul de l'indicateur les points d'arrêt associés au domicile, afin d'éclairer spécifiquement les pratiques spatiales des individus, i.e. les lieux réellement fréquentés en dehors de leur domicile.

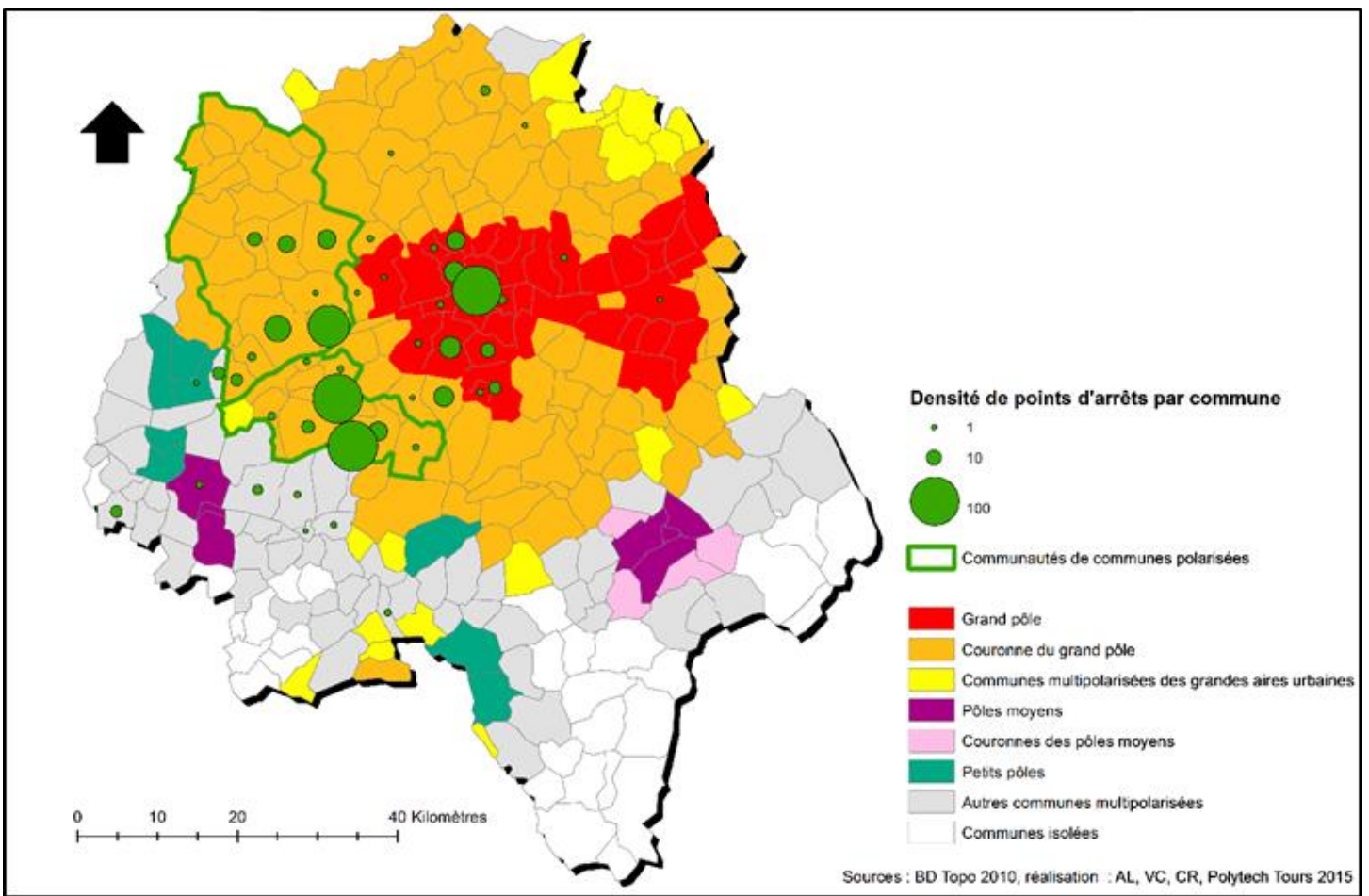

Carte 2 : Densité de points d'arrêt par commune d'Indre et Loire, selon le ZAU 2010, pour l'échantillon des habitants du rural polarisé - AL, VC, CR, Polytech Tours 2015

Il serait également intéressant de caractériser les territoires habités par $\mathrm{d}^{\prime}$ autres indicateurs, en particulier par le niveau d'équipements des communes. Nous avions en effet souhaité investiguer l'hypothèse selon laquelle le choix des territoires fréquentés par les individus serait guidé par leur niveau d'équipement.

Néanmoins, dans les territoires ruraux étudiés ici, la base permanente des équipements de l'INSEE ne permet pas de géolocaliser les équipements, ou très peu d'entre eux, et ne propose qu'une mesure de densité d'équipements par commune. Nos tentatives de croiser la densité de points d'arrêt et la densité d'équipements pour chaque type de territoire n'a pour le moment pas permis d'établir un lien explicite 
entre ces variables. Par ailleurs, pour cet indicateur, il serait une nouvelle fois judicieux de supprimer des points d'arrêts, ceux liés au domicile des individus, pour n'analyser que les autres lieux pratiqués.

\section{Indicateurs d'analyse temporelle}

On l'a mentionné à plusieurs reprises, l'un des intérêts des GPS par rapport aux carnets de pratiques, outre la précision des données géographiques sur les pratiques de mobilité, réside dans l'apport d'informations temporelles. Il est dès lors possible d'en tirer des analyses sur les heures de déplacements, les temps d'activité, les temps de mobilité/immobilité, d'opérer des comparaisons semaine/week-end ou jour/nuit par exemple.

A partir de la base de données de points d'arrêt, leur nombre et leur localisation, nous avons donc exploité la donnée complémentaire qu'est la durée passée dans chaque point d'arrêt: nous avons sommé les durées associées à l'ensemble des points d'arrêt des individus, pour l'ensemble des individus du rural polarisé puis de l'isolé, pour chaque commune fréquentée.

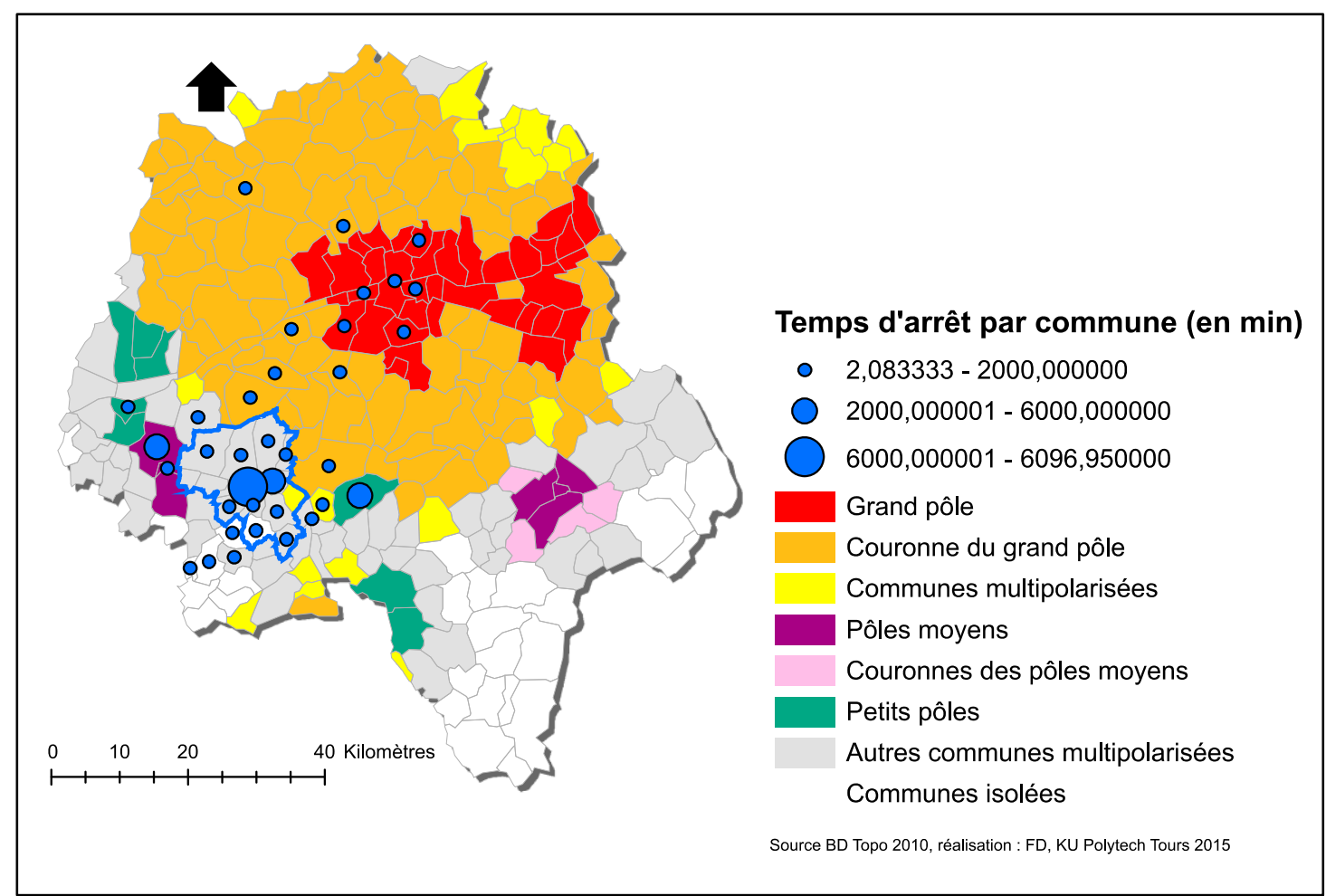

Carte 3 : Cumul des temps d'arrêt par commune d'Indre et Loire, selon le ZAU 2010, pour l'échantillon des habitants du rural isolé - FD, KU, Polytech Tours 2015 
Par rapport à l'indicateur basé sur la densité de points d'arrêts, celui-ci permet d'aller plus loin dans la compréhension des pratiques suivant le type de territoire. De fait, cet indicateur permet par exemple de mettre en évidence une différence notable dans la fréquentation du grand pôle par les individus suivis : alors que nous avions déjà noté une densité de points d'arrêts plus importante pour les habitants du rural polarisé, on peut en plus observer qu'ils y passent plus de temps que les habitants de l'isolé. A contrario, les individus résidant dans le rural isolé, s'ils pratiquent également le grand pôle, y restent peu de temps, en comparaison au temps passé dans le pôle moyen le plus proche (Chinon) et les petits pôles.

Le poids du motif travail est vraisemblablement structurant dans le constat que nous établissons pour les habitants du rural polarisé. Afin d'améliorer l'indicateur, il serait donc pertinent de segmenter les durées d'immobilité pour distinguer le temps du travail et celui passé au domicile, des temps consacrés aux autres motifs (loisirs, achats, etc.) ; ceci permettrait de spatialiser plus précisément l'intensité des pratiques moins ou non contraintes. Toutefois, la mise en œuvre est complexe, car si les lieux de résidence sont connus, les lieux de travail ne le sont pas aussi précisément (en particulier pour les actifs qui ont plusieurs lieux de travail). Ce manque de connaissance plaiderait pour que le protocole de suivi GPS soit accompagné d'une fiche descriptive détaillée de l'individu volontaire et de son mode de vie (horaires et amplitude de travail, etc.). Ces informations, qui pourraient être recueillies à l'occasion de la remise des traceurs GPS, engendreraient en revanche un alourdissement du protocole pour le volontaire, qui devrait ainsi se soumettre à un entretien, alors même que notre parti-pris a été au contraire d'alléger autant que possible nos sollicitations à son encontre.

\section{Exploitation cartographique}

Au-delà des approches qui visent à analyser les pratiques spatiales à travers des indicateurs synthétiques et statistiques, un autre intérêt des technologies GPS est la possibilité de représenter sous forme cartographique l'ensemble des données récoltées ; les relevés GPS, grâce à leur précision et à leur exhaustivité, permettent de visualiser une image-trace très proche de la réalité des pratiques spatiales des individus enquêtés.

Si l'analyse visuelle ne saurait constituer une mesure aussi précise et fiable que l'application d'un ensemble d'opérations mathématiques sur un jeu de données, elle n'en permet pas moins d'évaluer et d'analyser différents aspects qui peuvent s'avérer complémentaires des mesures statistiques. Bien que chronophage et nécessairement imparfaite (manque d'information sur les motifs des pratiques spatiales), l'analyse visuelle basée sur un langage graphique « naturel » qu'est la carte et facilement compréhensible permet une découverte synthétique des informations et 
la construction de connaissances intuitives et d'hypothèses, qui pourront être confirmées ou infirmées par l'analyse statistique ou par des entretiens semi-directifs.

De même qu'avec les indicateurs statistiques, on cherchera par cette analyse visuelle à analyser l'emprise des pratiques spatiales des individus, la forme générale de leur inscription territoriale ; on pourra également temporiser ces représentations selon différentes échelles (hebdomadaire, quotidienne, voire en temps réel, grâce à une représentation animée des pratiques); enfin, on pourra représenter de façon distincte l'information relative au mouvement (traits continus) et celle relative à l'immobilité ou à l'ancrage (ronds de surface proportionnelle à la durée des temps d'arrêt).

A titre exploratoire, nous avons souhaité mettre en œuvre un traitement synchronique (ensemble des pratiques sur une semaine) et désagrégé (pour chaque individu) des pratiques spatiales, en les classant selon les types de territoires ruraux de résidence. De ce traitement opéré sur l'ensemble des 57 traces, nous avons notamment $\mathrm{pu}$ faire ressortir différentes figures de l'inscription territoriale des pratiques spatiales.

\begin{tabular}{|c|c|c|c|}
\hline $\begin{array}{l}\text { Figures de } \\
\text { l'inscription } \\
\text { territoriale }\end{array}$ & $\begin{array}{l}\text { Type } \\
\text { d'investissement de } \\
\text { l'espace rural }\end{array}$ & $\begin{array}{l}\text { Type } \\
\text { d'investissement de } \\
\text { l'espace de la mobilité }\end{array}$ & $\begin{array}{l}\text { Type } \\
\text { d'investissement du } \\
\text { centre urbain }\end{array}$ \\
\hline Polarisé & Absence de pratique & Mobilité ponctuelle & Pratique intensive \\
\hline Territorialisé & $\begin{array}{l}\text { Pratique d'un espace } \\
\text { local élargi }\end{array}$ & Mobilité étendue & Pratique intensive \\
\hline Multipolarisé & $\begin{array}{l}\text { Pratique d'un espace } \\
\text { local élargi }\end{array}$ & Mobilité étendue & Absence de pratique \\
\hline
\end{tabular}

Figure 5 : Représentations cartographiques et caractéristiques des figures de l'inscription territoriale des pratiques spatiales dans les espaces ruraux polarisés étudiés - B. Feildel, 2016

Ces figures permettent d'identifier, et de poser pour hypothèse, l'existence de types différenciés d'investissement des espaces ruraux, des espaces de mobilité et des 
espaces urbains. Ces types contribuent ainsi à former des figures particulières, possiblement communes à un ensemble d'individus, de l'inscription territoriale des pratiques spatiales. Loin d'épuiser la diversité des situations rencontrées, ces différentes figures n'en permettent pas moins d'entrevoir et de confirmer la complexité des systèmes et des arrangements spatiaux qui composent les modalités de l'inscription territoriale des pratiques spatiales, entre ancrage et mobilité, dans les espaces ruraux.

Pour prolonger cette typologie cartographique, une piste de recherche envisagée vise à explorer des outils issus notamment de la théorie des graphes afin de définir de manière automatisée ces "patrons ", sur la base des image-traces GPS et de travaux récents impliquant notamment des chercheurs en informatique de Polytech Tours (Devogele et al., 2013 ; Etienne et al., 2015).

\section{Conclusion et ouverture}

Cet article à visée méthodologique avait pour objectifs de revenir sur les apports d'un suivi-GPS pour des recherches ou enquêtes sur les mobilités individuelles. Nous n'avons que très rapidement évoqué les résultats de la recherche en termes de connaissances et de compréhension des pratiques: nous renvoyons le lecteur au rapport final de la recherche (Aguiléra et al, 2016), ainsi qu'à la thèse de doctorat de M. Huyghe (2015) qui s'est spécifiquement intéressée à cette question.

Avant tout, concernant l'acquisition des données, notre expérience montre qu'il est préférable de paramétrer les appareils avec une fréquence d'enregistrement constante, quelle que soit la vitesse $\mathrm{du}$ déplacement: si une fréquence d'enregistrement variable n'empêche pas d'aboutir à des résultats cohérents, une même fréquence simplifie grandement l'interprétation (voire la permet, pour les déplacements de faible distance). Concernant la valeur de fréquence proprement dite, nous recommandons de la choisir en fonction d'objectifs clairement définis à l'étude : si celle-ci vise à travailler sur un territoire étendu comme celui de nos terrains d'étude, une fréquence assez faible (de l'ordre de 3-5 secondes) est possible pour s'adapter aux contraintes de capacité de mémoire des traceurs, mais dans ce cas, on saisit plus imparfaitement les déplacements très courts et/ou pédestres. A contrario, l'étude plus particulière de ces déplacements hectométriques exigera une fréquence plus forte, de l'ordre de 1 à 3 secondes.

Une première partie de nos résultats était consacrée au protocole mis en œuvre pour traiter les données à notre disposition : d'après la littérature, il semble en effet que les protocoles de traitement soient en évolution et souvent définis de manière indépendante par chaque équipe de recherche. Nous avons pour notre part utilisé le logiciel MobiRev, développé à l'Université de Tours : celui-ci n'a pas permis de supprimer, sans ambiguïté aucune, l'ensemble des points erratiques générés par 
les traceurs GPS ; il a en revanche été plus efficace pour identifier les points d'arrêts, sans pour autant aboutir à une parfaite certitude. Concernant les motifs de fréquentation de ces points d'arrêts, l'étude des traces-GPS seules n'est pas suffisante: nous recommandons donc que pour tout suivi GPS, la personne volontaire puisse remplir un carnet simplifié recensant les principaux lieux fréquentés durant la semaine et les motifs de déplacement. Si elle pourrait suffire dans la majorité des cas à lever les ambiguïtés, cette version très simplifiée des carnets de pratiques de type EMD s'ajouterait néanmoins au caractère déjà contraignant du traceur GPS. Il serait sinon envisageable de croiser les coordonnées des points d'arrêt avec les données des agendas électroniques des individus et/ou avec la géolocalisation des équipements lorsqu'elle est disponible sur l'INSEE, sur Google Earth ou d'autres bases de données comme par exemple OpenStreetMap ou la BDTopo de l'IGN.

Nous avons ensuite consacré une seconde partie de résultats à la présentation de différents types d'indicateurs d'analyse: indicateurs "synthétiques » issus des statistiques spatiales descriptives, indicateurs "spatialisés", indicateurs d'analyse temporelle et cartographique. Les résultats produits par ces indicateurs sont cohérents entre eux et avec les analyses tirées des entretiens semi-directifs (non présentées ici).

En termes méthodologiques, nous pouvons retenir que :

- pour caractériser l'étendue et la dispersion des territoires investis et pratiqués, nous avons développé deux indicateurs statistiques basés sur le calcul de distances cumulées, au domicile ou à un point central. Nous obtenons pour les deux indicateurs des résultats similaires, que les calculs soient réalisés à partir de l'ensemble des points enregistrés, ou à partir des seuls points d'arrêts. Dans cette optique, on pourrait donc éluder la phase d'identification des points d'arrêts, laborieuse et incertaine.

- deux autres types d'indicateurs (spatialisés et d'analyse temporelle) nous ont permis d'analyser les pratiques de mobilité en fonction des caractéristiques territoriales: nous avons analysé la densité de points d'arrêt et le temps d'immobilité dans les points d'arrêt en fonction d'une part du Zonage en Aires Urbaines de 2010 de l'INSEE, d'autre part de la densité d'équipements dans chaque commune. Pour obtenir des résultats probants, nous recommandons d'exclure de la base de points analysés ceux relatifs au domicile des individus et/ou à leur lieu de travail, pour éviter que ces derniers ne « masquent » les autres motifs.

- enfin, un dernier type d'analyse a été effectué à partir de représentations cartographiques des traces GPS, synchronique sur toute la semaine et désagrégée par individu : elle permet d'identifier une typologie des inscriptions territoriales, par type de territoire rural. Une piste de recherche envisagée vise, dans la 
continuité de ces analyses visuelles, à explorer des outils issus notamment de la théorie des graphes pour définir de manière automatisée ces "patrons ", sur la base des « image-traces » GPS et de travaux récents impliquant notamment des informaticiens de Polytech Tours.

Entre autres limites liées à l'utilisation des GPS, nous avons mentionné en introduction de cet article les questions éthiques posées par l'enregistrement de données à caractère personnel, qu'il est difficile d'anonymiser et qui peuvent permettre dans la plupart des cas d'identifier les individus suivis. Dans le cas des programmes MOUR et MOBITER, ces aspects éthiques n'ont pas été problématiques : nous avons simplement informé les volontaires des analyses qui seraient produites à partir de leurs traces, et fait une déclaration à la CNIL.

Nous approfondissons actuellement ces questionnements déontologiques et éthiques au cours d'un nouveau programme de recherche, qui aborde la question des Open data dans le cas de données à caractère personnel : nous nous intéressons aux problématiques soulevées par l'ouverture (mise à disposition des données en dehors du cadre de recherche dans lequel les données ont été produites) et l'analyse mutualisée de ce type de données (traces GPS, mais également entretiens semidirectifs); celles-ci posent des questions éthique et déontologique, mais également juridique et méthodologique.

\section{Références}

Aguilera A., Baptiste H., Feildel B., Huyghe M. \& Terral L. (2016). Rapport final Programme de recherche MOBIlité et dynamique des TErritoires Ruraux, $111 \mathrm{p}$.

Baptiste H., Busnot-Richard F., CARriere J.P., Huyghe M., MatTei M. (2013). Quelles mobilités en milieu rural à faible densité ? Rapport final du programme de recherche MOUR, $297 \mathrm{p}$.

BOURDIEU P. (1993). Effets de lieu, La misère du monde, Paris, Seuil, p. 159-167.

DAOUD R., GWIAZDZINSKI L. \& FILLOD-BARBARINO N. (2009). Le projet zigzag, un outil innovant pour observer les mobilités, Rapport de recherche, $6 \mathrm{p}$.

DePEAU S. \& QueVESSEUR E. (2014). A la recherche d'espaces invisibles de la mobilité, Usages, apports et limites des techniques GPS dans l'étude des déplacements urbains à l'échelle pédestre, Netcom Vol.28 (1-2), 35-54.

Devogele T., Etienne L., RAY C. (2013), Mobility Data: Modelling, Management, and Understanding, in RENSO C., SPACCAPIETRA S. \& ZIMANVI E. (Eds.) Part 3, Chapter 11: Maritime monitoring, 224-243, Cambridge University Press.

Drevon G., Gerber P., Klein O., EnAUx C. (2014), Intégration spatiale des frontaliers à travers les activités quotidiennes. Le cas de la région métropolitaine luxembourgeoise, Urbia - Les cahiers du développement durable, $\mathrm{n}^{\circ} 16,147-163$.

DREVON G. \& GWIAZDZINSKI L. (dir) (2014). Représenter des populations et des territoires en mouvement, Netcom Vol.28 (1-2).

Drevon G., KLEIN O. \& GWIAZDZINSKI L. (2015). Changement de regard sur les sentiers du quotidien : la vidéo géo-référencée pour mieux comprendre le déplacement, Colloque Temps, Art et Cartographie, Décembre 2014, Milan. 
Etienne L., Devogele T., Buchin M., Mcardle G. (2015). Trajectory Box Plot: a new pattern to summarize movements, International Journal of Geographical Information Science, Vol. 30 (5), 1- 19.

FEILDEL B. (2014). La mobilité révélée par GPS, Traces et récits pour éclairer les sens des mobilités, Netcom Vol.28 (1-2), 55-76.

Gerber P. \& CARpentier S. (2012). Impacts de la mobilité résidentielle transfrontalière sur les espaces de la vie quotidienne d'individus actifs du Luxembourg, INSEE, Economie et statistique $\mathrm{n}^{\circ} 157-158,77-96$.

GWIAZDZINSKI L. (dir) (2008). Le temps des adolescents, une approche spatio-temporelle des adolescents en périphérie de Besançon, Rapport d'étude à l'Union française des centres de vacances (UFCV), $89 \mathrm{p}$.

GwiazDZINSKI L. (2006). Déplacements des jeunes de Nanterre, Assises de la jeunesse, 4-10 décembre 2006

GWIAZDZINSKI L. \& KLEIN O. (2014). Du suivi GPS des individus à une approche chronotopique, Netcom Vol.28 (1/2), 77-106.

HuYGHe M. (2015). Habiter les territoires ruraux - Comprendre les dynamiques spatiales et sociales à l'œuvre, évaluer les perspectives d'évolution des pratiques de mobilité des ménages, Thèse d'Aménagement de l'espace et Urbanisme, Université de Tours, 464 p.

Huyghe M., Aguilera A., Baptiste H., Feildel B. \& Terral L. (2014). Programme de recherche MOBITER, Rapport intermédiaire $\mathrm{n}^{\circ} 1,160 \mathrm{p}$.

Martouzet D., Bailleul H., Feildel B., Laffont G.-H., Thibault S., 2012, « Le périurbain à l'épreuve des modèles d'habiter. La viabilité périurbaine entre théorie(s) et pratique(s) », Tours, Rapport de recherche PUCA, MEDD, 309 p.

NGUYEN D. (2012). Faisabilité d'une Enquête Globale Transports (EGT) intégrale par association d'un GPS, d'un SIG et d'un Système expert en Ile-de-France, Rapport final, PREDIT, 98 p.

Poirier G., SPILliaert S., Uguen Y.-M., Viannes Q. (2015), Pratique de la mobilité spatiale quotidienne en milieu rural, dir. Baptiste H., Feildel B., Huyghe M., Projet de Fin d'Etudes, Ecole Polytechnique de l'Université de Tours, Département Aménagement.

Primault V., Ben MoKhtaR S., Lauradoux C. \& BRUnie L. (2015). Time distortion anonymization for the publication of mobility data with high utility, 2015 IEEE Trustcom/BigDataSE/ISPA Vol.1, 539-546.

SERRA M. (2014). MobiRev : la mobilité révélée par GPS. Logiciel de traitement de traces GPS, Rapport de PFE Tours, Polytech Tours, $44 \mathrm{p}$. 
Annexe 1 : Limites administratives des 101 départements français

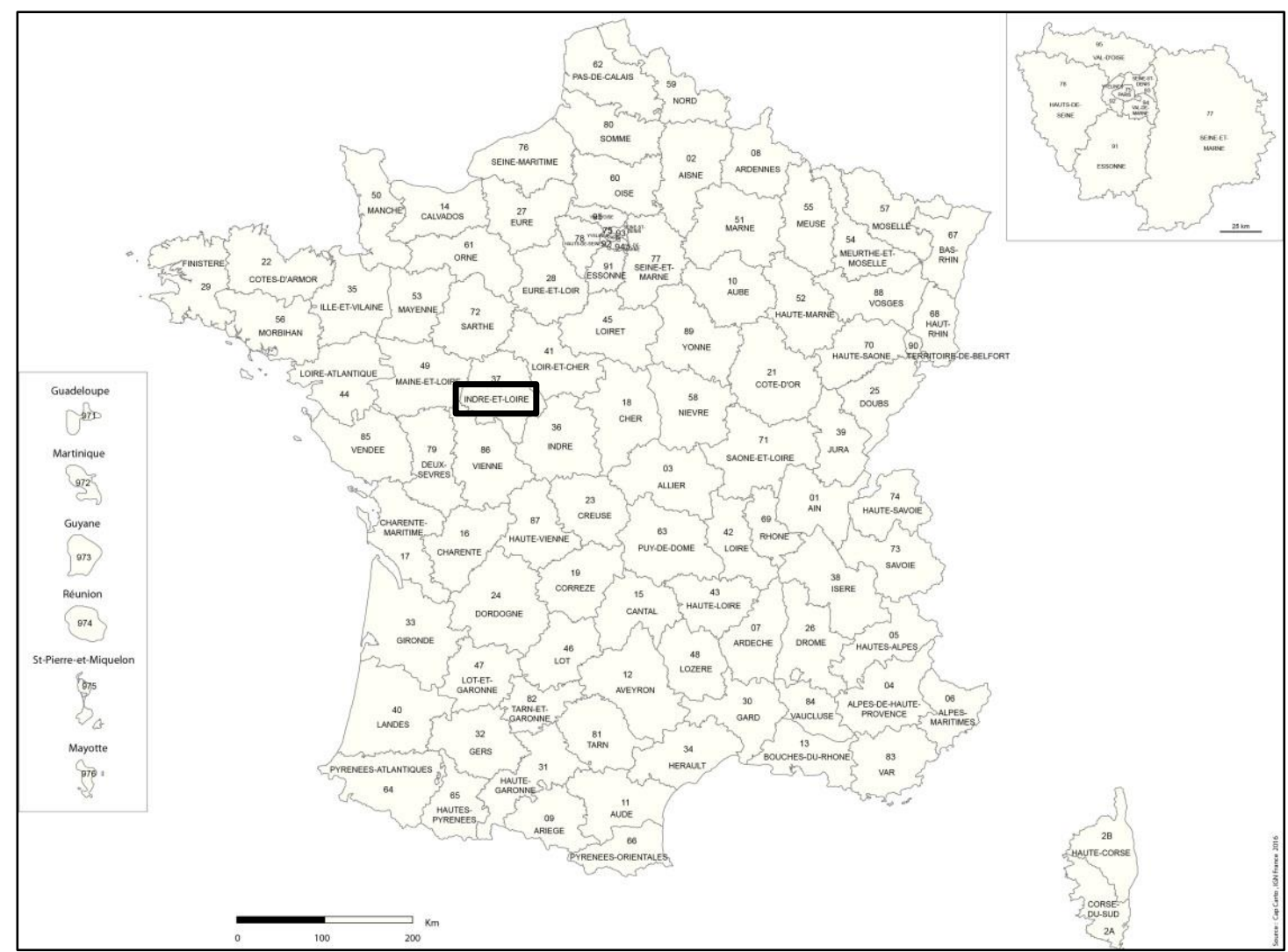

En France, le département est en premier lieu une circonscription administrative décentralisée de l'État, placé sous l'autorité d'un préfet, à partir d'une « capitale » (la préfecture). La France est ainsi subdivisée en 101 départements (dont 5 en outre-mer). C'est en second lieu une collectivité territoriale, administrée par un conseil départemental, dont les compétences ont été redéfinies en 2015 par la loi « Nouvelle Organisation Territoriale de la République ». Ces compétences ont trait notamment à l'action sociale, à la gestion des infrastructures routières départementales ou encore à celle de certains établissements scolaires (les collèges).

Par ailleurs, le département est lui-même constitué de regroupements de communes qui s'associent sous la forme d'Etablissements Publics de Coopération Intercommunale (EPCI), leur permettant d'exercer des compétences propres et d'élaborer un projet commun de développement et d'aménagement. Ces EPCI ont été redéfinis suite à la loi de "Modernisation de l'Action Publique Territoriale et d'Affirmation des Métropoles » en 2014.

L'Indre et Loire est un de ces départements français, situé dans le quart nord-ouest de la France, comprenant 272 communes et 604966 habitants en 2015 (INSEE), dont la préfecture est la commune de Tours. Ce département est caractérisé par une certaine « hypertrophie » de la métropole de Tours Val de Loire (intercommunalité de 22 communes regroupant 292268 habitants en 2015, dont 136252 résident à Tours), concentrant une part importante de l'emploi, des activités économiques et des équipements du département.

Les territoires d'études pour cet article couvrent 3 intercommunalités existantes durant les 2 projets de recherche MOUR et MOBITER, regroupant des communes dites rurales polarisées par la métropole de Tours, dans la communauté de communes de Touraine Nord-Ouest (CCTNO) et celle du Pays d'Azay le Rideau (CCPAR) et des communes dites rurales isolées dans la communauté de communes du Bourchardais (CCB). 